\title{
Transrenal Ureter Embolization for Refractory Urine Leaks from latrogenic Ureteric Injury Following Colorectal Surgery
}

\author{
1) Department of Radiology, Saiseikai Utsunomiya Hospital, Japan \\ 2) Department of Surgery, Saiseikai Utsunomiya Hospital, Japan \\ 3) Department of Urology, Saiseikai Utsunomiya Hospital, Japan
}

Koki Kato ${ }^{1)}$, Syunkichi Sasazawa ${ }^{1)}$, Takashi Hazama ${ }^{1)}$, Kei Ishimura ${ }^{1)}$, Keiichi Tanimura ${ }^{1)}$ Hiroharu Shinozaki ${ }^{2)}$, Ryou Tsukamoto ${ }^{3)}$, Toyofusa Tobe ${ }^{3)}$

\begin{abstract}
We present the cases of two patients who underwent ureteral occlusion using coils and/or Amplatzer Vascular Plug with N-butyl cyanoacrylate glue after extensive advanced rectal surgery. Both patients had complex urine leaks unresponsive to urinary diversion. In view of the progress of the disease and the history of polysurgery, reconstructive surgery or anterograde ureteral stent insertion was not chosen. All patients had immediate resolution of urinary leakage after ureteral embolization, resulting in symptom relief throughout the follow-up period. There were no procedure-related complications or side effects.

Key words: Urinary fistula, ureteral embolization, Amplatzer vascular plugs

(Interventional Radiology Advance Publication)
\end{abstract}

\section{Introduction}

Iatrogenic ureteric injuries are rare but serious complications of abdominopelvic surgery. The incidence of ureteric injury ranges from $0.05 \%$ to $30 \%$ in all pelvic surgery and is higher in patients with recognized risk factors. When identified intraoperatively, immediate repair and secured drainage results in fewer complications and superior outcomes $[1,2]$. However, $65 \%-80 \%$ of ureteric injuries are only detected postoperatively, and current guidelines recommend stent insertion or percutaneous nephrostomy (PCN). Delayed detection of ureteral trauma can progress with serious pathological conditions, such as fistula formation, sepsis, and renal dysfunction, often requiring additional treatment and long-term hospitalization.

The initial management strategy typically requires the diversion of urine away from the site of leakage with a large caliber nephrostomy from above and a Foley catheter from below, with or without a ureteral stent. When this fails, treatment can be difficult to achieve and life-altering for the patient. The surgical repair of these fistulas is often unsuccessful because of prior surgery and the inflammatory reaction caused by the urine leak, all of which has encouraged minimally invasive options. Ureteral embolization is considered one of the options for ureteral injuries that are difficult to treat [3-9].

The cases of two patients suffering refractory urine leaks from iatrogenic ureteric injury following colorectal cancer surgery treated by transrenal ureteral embolization are presented.

The procedures described in this article were approved by the institutional review board of our institution, and written informed consent was obtained from the patients for the publication of the details of their cases. 


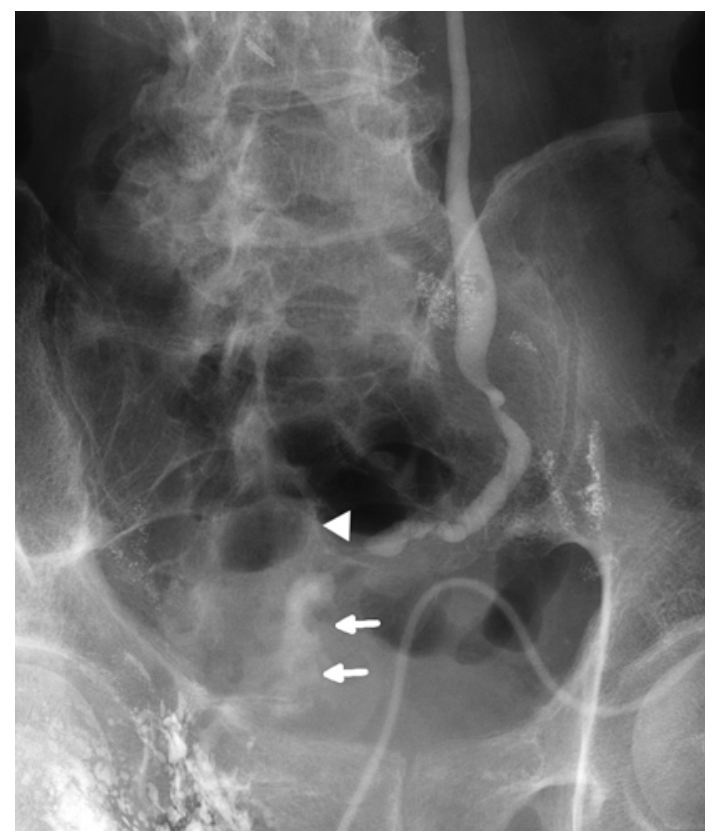

Figure 1. Urography shows complete ureteral transections (white arrowhead). An extraluminal contrast material (white arrows) is seen at the level of complete ureteral transection. Lipiodol accumulation in the bilateral pelvic lymph nodes is noted from previous lymphangiography.

\section{Case Reports}

\section{Case 1}

A 70-year-old man was diagnosed with colonic obstruction due to advanced rectal cancer with invasion to the seminal vesicles, and emergent ileostomy was performed. $\mathrm{He}$ then received neoadjuvant chemoradiotherapy consisting of concomitant radiation $(50.4$ Gy external beam radiation in 28 fractions) and neoadjuvant therapy with capecitabine (Xeloda, Roche Corp., France) $2500 \mathrm{mg} / \mathrm{m}^{2}$ concomitantly with radiation therapy. The laparoscopic Miles' operation was performed 3 months after the ileostomy.

After the Miles' operation, about $2000 \mathrm{ml}$ of drainage continued from the drain. The attending physician interpreted this as the effect of liver cirrhosis. Renal function slightly deteriorated from the second week after surgery. It was initially considered lymphorrhea on the basis of the experience of the attending physician, and lymphangiography was performed 2 months after the Miles' operation. However, leakage from the pelvic drain did not subside. The excretion phase of the contrast-enhanced computed tomography (CECT) 4 months after the Miles' operation showed the leakage of the contrast-enhanced urine in the right retroperitoneal space that extended to the space of the presacral region around the pelvic drainage tube, indicating ureteral injury. Retrograde ureterography was performed and showed obstruction of the right lower ureter. A urologist was unable to insert a hydrophilic guidewire into the right renal pelvis;

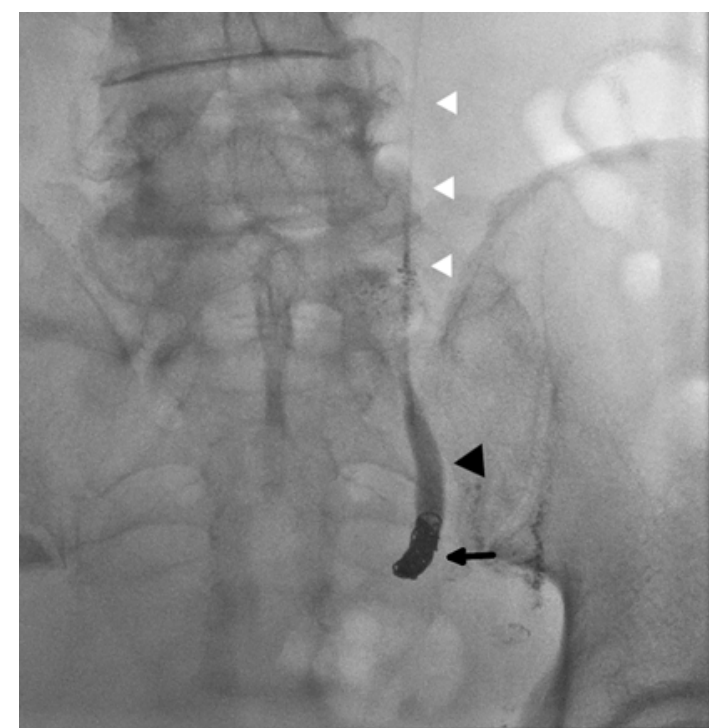

Figure 2. Fluoroscopic image shows coils (arrow) and NBCA glue (arrowhead) in the ureter. White arrowheads indicate the microcatheter.

however, ureteric stent insertion failed.

Therefore, urine diversion by nephrostomy tube placement was attempted, and urography showed complete ureteral transections and extraluminal contrast material at the level of complete ureteral transection (Fig. 1). However, drainage of $200 \mathrm{ml} /$ day or more continued. Symptomatic relief was not obtained by PCN.

For refractory urine leakage, the attending physicians explained permanent embolization of the ureter and nephrostomy to the patient and family and obtained consent for the treatments.

First, the nephrostomy tube was exchanged to a $16-\mathrm{F}$ sheath. The ureteroscope was inserted to the sheath and showed a fistula that reached to the space around the pelvic drainage tube at the upper sacral level. Then, a 5-F Berenstein tipped catheter (Imager II, Boston Scientific, Natick, MA, USA) was inserted through the sheath, and the fistula was confirmed under ureterography. Embolization was performed just above the fistula using two interlocking detachable coils $(8 \mathrm{~mm} / 20 \mathrm{~cm} \times 1,6 \mathrm{~mm} / 20 \mathrm{~cm} \times 1)($ Boston Scientific, Tokyo, Japan).

A microcatheter (Progreat $\beta 3$, Terumo, Tokyo, Japan) was then advanced just above the coil, $5 \mathrm{ml}$ of autologous blood were injected to promote polymerization of N-butyl-2cyanoacrylate (NBCA; Histoacryl; Braun, Tuttlingen, Germany), and NBCA mixed with Lipiodol (Guerbet, Villepinte, France) at a ratio of 1:1 was injected through the microcatheter (Fig. 2).

When the microcatheter was removed, NBCA adhesion occurred at a 1.9-F microcatheter tip, and part of the NBCA cast adhered to the catheter and moved upstream. However, the fistula disappeared on the final urography (Fig. 3). The sheath was replaced with a nephrostomy catheter, and the procedure was completed. No complications were seen after embolization, and drainage volume decreased to less than 10 


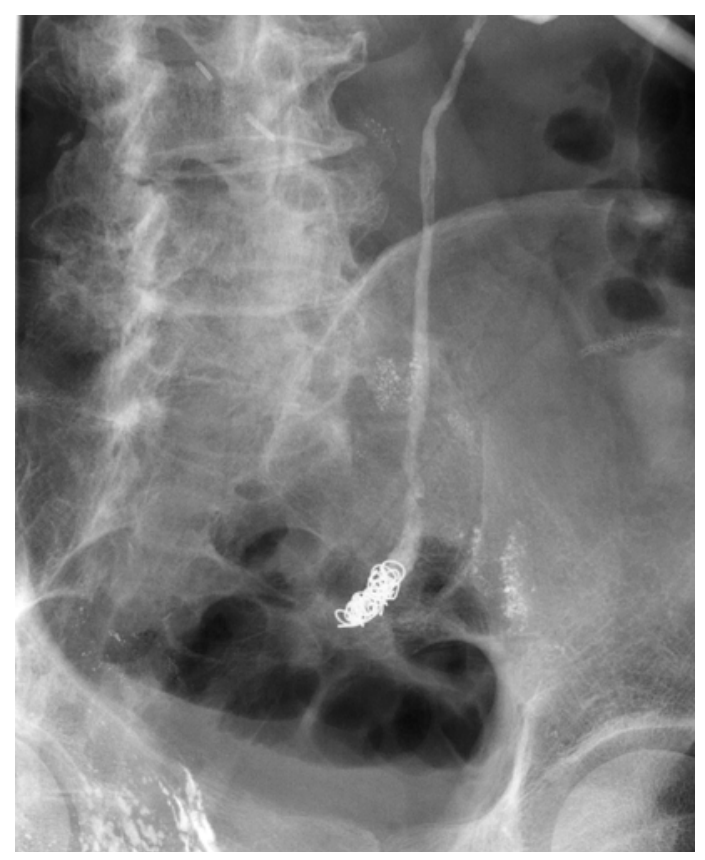

Figure 3. Pyelogram demonstrates complete ureteral occlusion with the coils and NBCA glue.

$\mathrm{ml} /$ day. The drain was removed 3 weeks after ureteral embolization. Rectal cancer recurrence appeared on the pelvic floor during a 3-year follow-up, but no recurrence of urinary leakage was observed.

\section{Case 2}

A 40-year-old man was diagnosed with stage IIIa rectal cancer and underwent laparoscopic low anterior resection and ileostomy. He was started on adjuvant chemotherapy with capecitabine after the first surgery for 6 months. Colostomy closure was performed 1 year after surgery. For the disseminated lesions at the lower abdominal wall 2 years after the first operation, he underwent resections of the disseminated lesions and partial proctectomy. After resection of the recurrences, the patient complained of fever and left lower abdominal pain. A CECT scan demonstrated leak of contrast material from the left ureter to a retroperitoneal collection. On postoperative day 24 , retrograde ureteropyelography showed left ureteral injury, and the urinoma was not opacified. A urologist was unable to insert hydrophilic guidewires into the left renal pelvis to allow urine drainage. On postoperative day 28, a urologist tried to insert an anterograde ureteral stent, but the guidewire did not pass, and only PCN was performed. A pyelogram demonstrated complete ureteral transections and a focal outpouching of the extraluminal contrast material at the level of complete ureteral transection (Fig. 4). Two weeks later, follow-up CECT showed a persistent retroperitoneal urinoma. However, drainage volume did not decrease below $200 \mathrm{ml}$ per day.

Because of the early resumption of chemotherapy, the surgeons and urologists requested ureteral embolization. In view of the progress of the disease and the history of polysurgery, the surgeons and urologists judged that reconstruc-

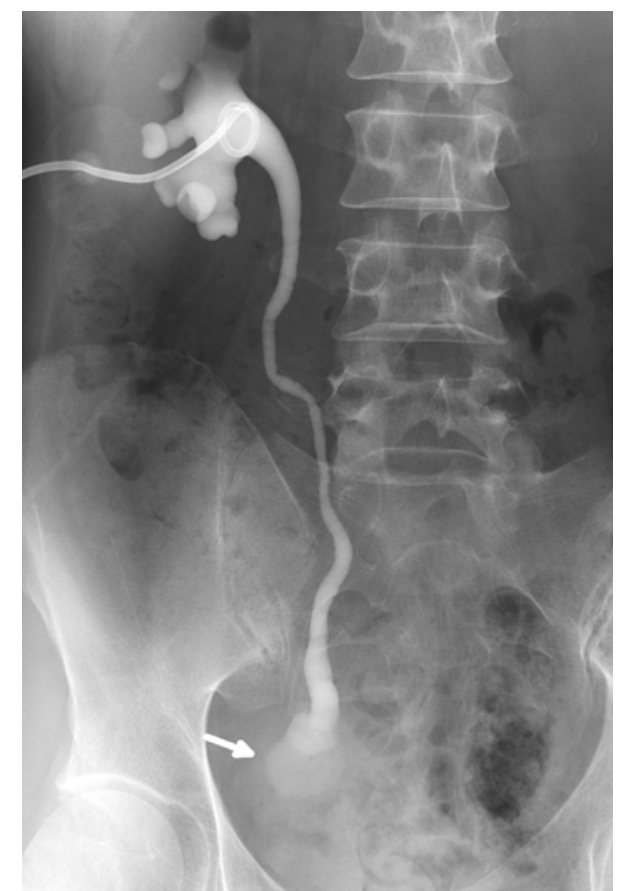

Figure 4. Pyelogram shows complete ureteral transections. A focal outpouching of extraluminal contrast material (white arrow) is seen at the level of complete ureteral transection.

tive surgery or anterograde ureteral stent insertion did not seem justified. The attending physician explained to the patient the treatment policy, aiming for early resumption of chemotherapy by sacrificing the kidney, and the patient agreed. At this point, the surgeons and urologists requested ureteral embolization.

The procedure was performed under sedation with dexmedetomidine hydrochloride (Precedex, Hospira, Lake Forest, IL, USA). First, a nephrogram was obtained, and the renal pelvic ureter and urinoma were confirmed. The nephrostomy catheter was replaced with a 6-F guiding sheath (Destination, Terumo, Japan) using a 0.035-inch guidewire (Terumo, Tokyo, Japan). A 4-F Tempo catheter (Cordis, Tipperary, Ireland) was inserted into the sheath and advanced to the lower ureter using a guidewire. The sheath was placed on the catheter, and the tip was advanced to the lower ureter. A 7-mm-diameter Amplatzer Vascular Plug II (AVP II) (St. Jude Medical, St. Paul, MN) was then released.

Furthermore, a microcatheter (Carnelian Pixie, 1.8F/2.7F $135 \mathrm{~cm}$, Tokai Medical Products, Aichi, Japan) was inserted, and embolization proceeded with an $8 \mathrm{~mm} / 20 \mathrm{~cm}$ length coil (Interlock 2D, Boston Scientific Corporation, Cork, Ireland). Embolization with NBCA-Lipiodol mixture in a ratio of 1:2 was performed (Fig. 5). Urinomas were not visible on the nephrogram after embolization (Fig. 6). Finally, using a guidewire, the sheath was replaced with an 8-F JINRO TM pigtail nephrostomy catheter (Boston Scientific Corporation) to complete the procedure. Two weeks after ureteral embolization, the nephrostomy tube was clamped.

Although the patient had a slight fever, his condition improved with antibiotics and an antipyretic agent after one 


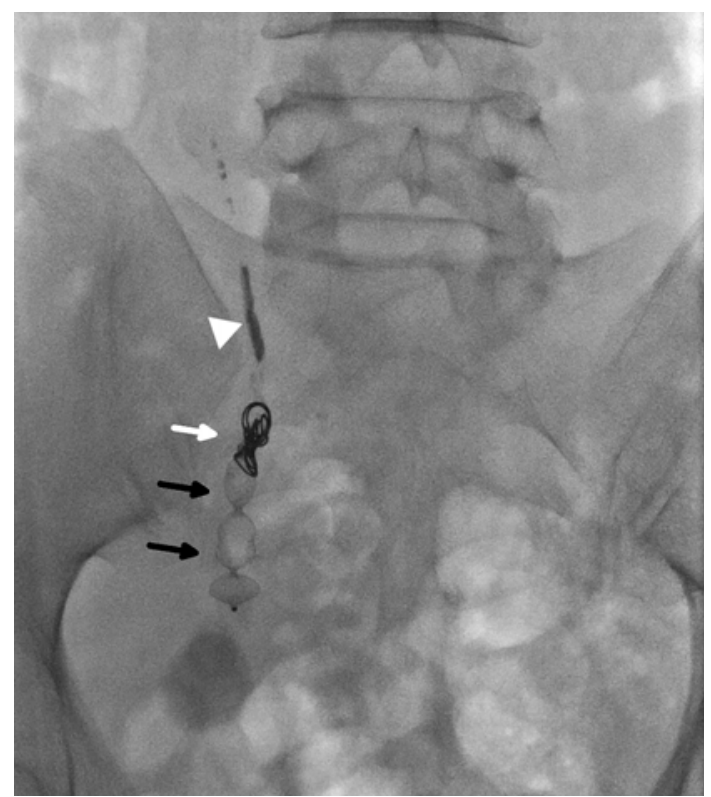

Figure 5. Fluoroscopic image shows the plug (arrows), coil (white arrow), and NBCA glue (white arrowhead) in the ureter.

week. Three months after ureteral embolization, CT showed disappearance of the urinoma. Renal dysfunction after ureteral embolization remained mild. Therefore, the nephrostomy tube was removed. The patient continued chemotherapy, and no recurrence was seen during a 2-year follow-up.

\section{Discussion}

Conservative and surgical management of a fistula of the lower urinary tract fails in as many as $35 \%$ of cases $[3,4]$. These patients are frequently poor surgical candidates and are often initially treated with urinary diversion via PCN tubes. For patients with persistent urinary leakage, transrenal ureteral occlusion with permanent urinary diversion may be performed as a palliative treatment $[3,4]$. That is, $\mathrm{PCN}$ and ureteral stent insertion are the first treatment of choice for ureteral injury. However, if it is technically difficult or at high risk, ureteral embolism may be a treatment option.

Given the mechanism and pathogenesis of efficient prolonged occlusion of the ureters, agents that stimulate the urothelium to induce hyperplastic tissue formation and ureteric strictures should be administered [5]. Various techniques with coils and gelatin sponges, isobutyl-2cyanoacrylate, detachable balloons, nylon plugs and bare and covered stents, Gianturco coils with or without gelfoam pledgets (Pharmacia \& Upjohn, Kalamazoo, MI, USA), percutaneous clips, nondetachable and detachable balloons, tissue adhesive, electrocautery, and silicone occluding devices have been described [3-9]. Stainless steel coils with Dacron fibers, AVP, and metal stents can cause such irritative effects on the urothelium, which explains the paradoxical use of stents as a method to occlude the ureter [3-6].

Kim et al. reported five patients with ureterovaginal fistu-

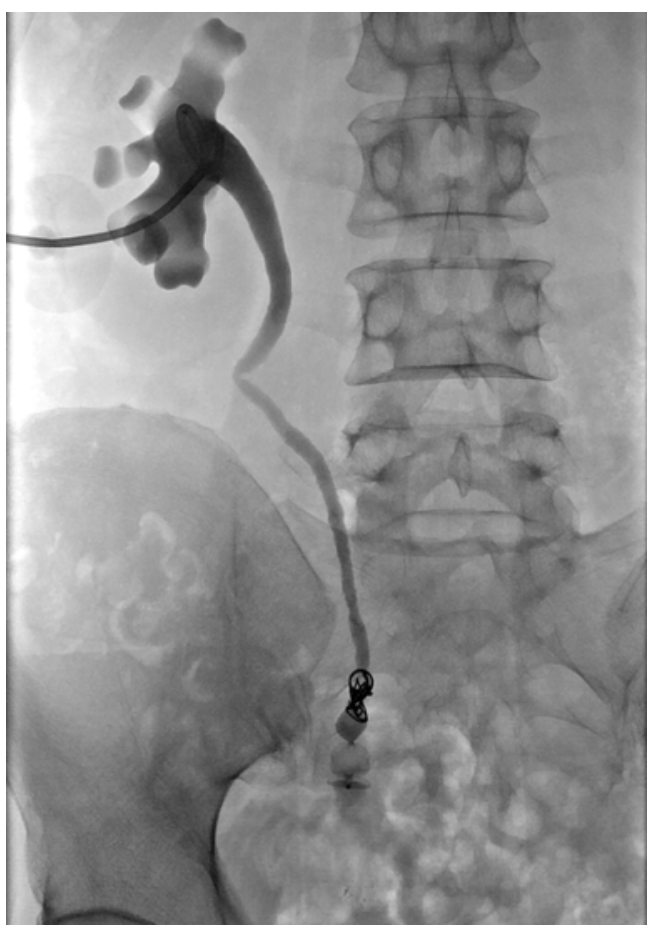

Figure 6. Pyelogram demonstrates complete ureteral occlusion by the plug, coil, and NBCA glue.

las who were technically successfully treated with transrenal ureteral occlusion with microcoils. Complete or near complete $(<1 \mathrm{pad} /$ day $)$ dryness was obtained in all patients within 3 day. No complications other than slight proximal migration of the microcoils occurred in two patients. The PCN tubes were removed in four of the five patients during the follow-up period with the following procedures: antegrade ureteral stent placement, patent normal ureter, a ureteroneocystostomy, and a laparoscopic end-to-end anastomosis of the ureter [7].

The occlusive nature of the AVP, a wire mesh with patent interstices, in the vascular system relies on the hemostatic mechanisms of the vascular system. Therefore, the AVP would not acutely occlude the ureter. Schild et al. described a case of distal ureteric embolization using an AVP placed in a latex "envelope" (condom tip) to make the AVP impermeable to urine [6].

Huber et al. reported nine consecutive female patients who underwent ureteral embolization via a "sandwich" technique with two vascular plugs and NBCA glue for ureteral fistulae unresponsive to urinary diversion. In seven patients (78\%), urinary leakage immediately resolved, and in the remaining two (22\%), unilateral repeat treatment was required to eliminate leakage. In all patients, symptom resolution lasted throughout the follow-up period [4].

Liquid embolic agents, such as NBCA, show a high rate of immediate occlusion. Nouri et al. reported that the injection of NBCA is an important factor for a successful urinary fistula closure. NBCA is a strong tissue-adhesive material, and they expected to seal the wall of the urinoma cavity and consequently obliterate the space of urine accumulation even 
in a case of urinary leakage without a distinct fistulous tract [8]. However, with liquid embolic agents, the glue commonly softens, migrates, fragments, and/or recanalizes with time $[5,6]$. They are often inferior to coils or metallic occlusion devices for long-term occlusion [5].

In the present case, it was decided to use NBCA as the short-term embolic agent deployed upstream of AVP or coils, which serve to contain the glue and inhibit migration. In this method, the glue provided an acute occlusive effect, whereas the AVP or coils, aside from entrapping the glue, served as a urothelial irritant to cause scarring and, ultimately, long-term ureteral occlusion.

Nephrostomy was performed in both cases. Therefore, radiologists proposed antegrade ureteral stent placement or ureteral stent placement using the rendezvous technique attempted via antegrade and retrograde approaches. However, urologists and surgeons feared that the condition could be exacerbated by manipulating guidewires and catheters in the injured area of the ureter. Therefore, we decided to perform ureteral embolization.

There are several other methods that might sacrifice renal function. For example, renal artery embolization might be useful in stopping urinary leakage; however, it is often accompanied by postembolic pain. In our cases of iatrogenic ureteral injury, we chose to avoid painful treatment.

In Case 2, the PCN tube was removed after ureteral embolization. From the perspective of preserving renal function, using a permanent PCN was desirable. However, the patient was young and emphasized quality of life; thus, it was decided to remove the PCN.

Ureteral embolization has many drawbacks, such as continuous urinary flow through the "obstructed" site, coil dislodgment, softening of obstructing material, and recanalization of occlusion. However, ureteral diameter varies with peristalsis; therefore, in the absence of standard sizing guidelines, the size of the plug was left to the discretion of the operator in the previous study. Schild et al. [6] reported that the AVP retains its elasticity over time, and the migration of the occluding material, such as that seen with detachable balloons or coils, should be less of a problem, since the device may adapt to varying ureteral diameters.

Because of the off-label use of the AVP and the NBCA glue and Lipiodol mixture, there were no guidelines on the appropriate glue/Lipiodol ratio, volume of administered liquid embolic agent, and sizing of plugs within the ureter [9]. In fact, at the time of Case 1, we still did not use a plug for ureteral embolism because of the off-label use.

Huber et al. reported a case with bilateral internal iliac artery pseudoaneurysms that developed adjacent to the plugs 5 months after ureteral embolization. The patient had a history of radiation therapy, and the plugs had been placed at and slightly below the level of the pelvic brim. In the case of bilateral iliac artery pseudoaneurysms, the plugs were oversized by two or three times the diameter of the ureter. Other patients underwent embolization with similarly sized plugs without the development of pseudoaneurysms. They suggested that placing the plugs above the pelvic brim and choosing plugs with less aggressive oversizing may help alleviate serious complications [4].

In summary, two cases of successful transrenal ureteral occlusion using an AVP or detachable coil with NBCA glue were presented. Transrenal ureteral occlusion is a safe and reliable method. We believe that this technique merits further investigation.

\section{Conflict of Interest: None}

\section{References}

1. Kitrey ND, Djakovic N, Hallscheidt P, et al. EAU guidelines on urological Trauma 2020. Cited 2011. https://uroweb.org/guideline/ urological-trauma/.

2. Elliott SP, McAninch JW. Ureteral injuries: external and iatrogenic. Urol Clin North Am. 2006; 33: 55-66.

3. Shindel AW, Zhu H, Hovsepian DM, Brandes SB, Shindel AW. Ureteric embolization with stainless-steel coils for managing refractory lower urinary tract fistula: a 12-year experience. BJU Int 2007; 99: 364-368.

4. Huber T, Wilkins LR, Sabri S, et al. Midterm follow-up of ureteral embolization using vascular plugs and N-butyl cyanoacrylate glue. J Vasc Interv Radiol 2017; 28: 1123-1128.

5. Saad WE, Kalagher S, Turba UC, et al. Ureteric embolization for lower urinary tract fistulae: use of two Amplatzer vascular plugs and N-butyl cyanoacrylate employing the "sandwich" technique. Cardiovasc Interv Radiol 2013; 36: 1068-1072.

6. Schild HH, Meyer C, Möhlenbroch M, Mueller SC, Simon B, Kuhl CK. Transrenal ureter occlusion with an Amplatzer vascular plug. J Vasc Interv Radiol 2009; 20: 1390-1392.

7. Kim SK, Lee YR, Kyung MS, Choi JS. Transrenal ureteral occlusion with the use of microcoils in five patients with ureterovaginal fistulas. Abdom Imaging 2008; 33: 615-620.

8. Nouri YM, Chu HH, Shin JH, et al. Percutaneous obliteration of urinary leakage after partial nephrectomy using N-butylcyanoacrylate obliteration of the urinoma with or without coil embolization of the fistula tract. J Vasc Interv Radiol 2019; 30: 20022008.

9. Jin MX, Mohabir AD, Caplin DM, Lobko I, Siegel DN. Transrenal ureteral embolization utilizing Amplatzer vascular plugs and N-butyl cyanoacrylate glue. J Endourol Case Rep 2018; 4: 108-110.

Interventional Radiology is an Open Access journal distributed under the Creative Commons Attribution-NonCommercial 4.0 International License. To view the details of this license, please visit (https://creativecommons.org/licenses/bync/4.0/). 